\title{
Low Cost UWB X-Band LFMCW-SAR
}

\author{
Tao Lai*, Ke Jin, Tao Hu, Gong-quan Li, Yong-jun Zhao \\ * Zhengzhou Institute of Information Science and Technology, \\ Zhengzhou, China,ltnudt@163.com
}

Keywords: LFMCW-SAR; YIG Oscillator; Nonlinearity Correction; Algorithm.

\begin{abstract}
For near range high resolution SAR (synthetic aperture radar) imaging, this paper presents an scheme for generating X-band LFMCW signal with $5.1 \mathrm{GHz}$ bandwidth based on YIG oscillator. The method is simple in structure and low in price, but introduces a certain degree of nonlinearity, resulting in range-resolution degradation. This paper first introduces the design of the radar transmitter. After that, with the existing HAF-based (high-order ambiguity function) nonlinearity correction method and $\omega k$ imaging algorithm, well focused range profiles and SAR images are obtained. Measurement results are used to validate the effectiveness of the algorithm. In the end, several SAR images are given to shown the performance of the radar system.
\end{abstract}

\section{Introduction}

Compared with the pulse radar, linear frequency modulated continuous wave (LFMCW) radar has the advantages of lightweight, low cost and low probability of intercept, which has played an important role in target detection. Using the deramp-on-receive (dechirp) operation, LFMCW radar can collect the signal with low sampling rate even under large instantaneous bandwidth. Therefore, lots of short range imaging radar systems with huge bandwidth are based on the dechirp mechanism of LFMCW. For example, Kansas University (USA) has developed an S/C-band array radar (with $6 \mathrm{GHz}$ bandwidth) to detect the depth of snow [1]. Northeastern University (USA) has designed a W-band person-borne explosive detection radar with $8 \mathrm{GHz}$ bandwidth [2]. The W-band LFMCW radar (with $25.6 \mathrm{GHz}$ bandwidth) developed by Ruhr-University Bochum (Germany) [3] has implemented radar imaging of gun on human body successfully. Furthermore, some Sub-THz radar systems (with bandwidth above $40 \mathrm{GHz}$ ) emerged in Ruhr-University Bochum [4] and Nanyang Technological University (Singapore) [5] to implement short range detection.

In this paper, the results of an X-band LFMCW radar system development and signal processing are reported. This radar system is a short range detection sensor with homodyne architecture, which has the advantage of simple configuration and short development period. With the bandwidth of $5.1 \mathrm{GHz}$, a high range resolution can be achieved. When put on a rail and driven by a motor, the radar can conduct two dimensional imaging. However, the problem of VCO (Voltage Controlled Oscillator) nonlinearity deteriorates range resolution seriously by spreading the target energy through different frequencies [6]. Later, this paper will mainly introduce the design of the transmitter and receiver under the conditional of large bandwidth. At the same time, aiming at the problems of modulation nonlinearity and synthetic aperture radar (SAR) imaging, HAF-based (high-order ambiguity function) nonlinearity correction method [7] and $\omega k$ algorithm [8] are adopted respectively. The algorithms above are tested with a number of range profiles and SAR images. Finally, copper cylinder and model airplane images are given to demonstrate the performance of this X-band radar system.

\section{Transmitter architecture}

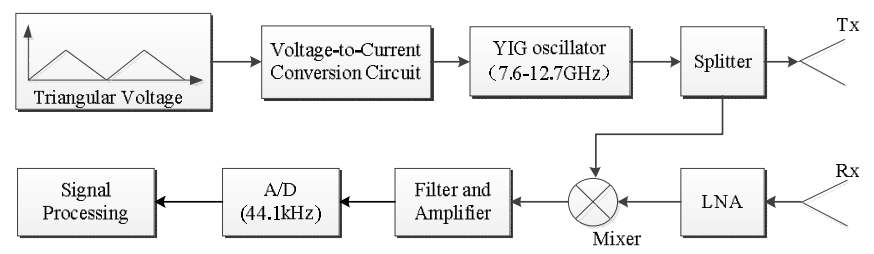

Fig. 1 Block diagram of the X-band FMCW radar.

The block diagram of the X-band LFMCW radar system is shown in Fig.1. To simplify the process of system design, the homodyne architecture is applied in this FMCW radar, which means the echo signal is directly mixed with the transmitted signal. In the signal source module, the YIG (Yttrium Iron Garnet) oscillator is chosen out of two reasons. First, a high linearity of frequency versus current can be obtained. Second, flat amplitude-frequency characteristics of YIG oscillator can be ensured in a wide range of frequencies, which is about $\pm 2 \mathrm{~dB}$. These two advantages of the oscillator insure the quality of the wideband transmitted signal to a certain degree, so that a well compressed range profile is expected. Instead of the PLL (Phase-locked loop), the structure of YIG is employed, driven by linear triangular voltage to generate LFMCW signal. The driving voltage can be obtained with the DDS (Direct Digital Synthesizer) method thanks to its excellent linearity.

However, different from the VCO, the output frequency of YIG is determined by the input current, which is usually several hundred milliampere. In this system, we use the AV783 oscillator whose nominal working frequency is $8 \sim 12.4 \mathrm{GHz}$. The tuning end of the oscillator equals to a series connection of a $9 \Omega$ resistance and a $60 \mathrm{mH}$ inductance.

The tuning circuit of the YIG oscillator is shown in Fig.2. To obtain the signal of $7.6 \sim 12.7 \mathrm{GHz}$, the tuning current should be $370 \sim 640 \mathrm{~mA}$. Therefore, the driving linear triangular voltage should be $1.48 \sim 2.56 \mathrm{~V}$, with the sampling resistance $R 8=4 \Omega$. In the left side of Fig. 2 , the input triangular wave generated by the DDS is $0 \sim 3.3 \mathrm{~V}$. So, this triangular wave should be scaled and dc offset. In the following, the 
amplifierU2A was used to implement the addition function, which added the adjustable dc voltage and the adjustable scaled triangular wave. The output triangular wave was used to drive the sampling resistance $\mathrm{R} 8$ to get the needed tuning current. With small input impedance, the YIG oscillator can't be driven by the output triangular wave. A voltage-to-current switching circuit is needed later. In Fig.2, the Darlington transistor Q1 and amplifier U1A were utilized to make up a typical switching circuit. Thanks to the high driving capability of Darlington transistor and almost same current value in its emitter and collector, the input current of YIG will be the same with that in R8. In this way, the linear frequency modulated (LFM) signal was obtained. As measured, the linearity of the signal was about $2.2 \%$. In the homodyne architecture, the wideband signal was then amplified and mixed with the local oscillator (LO) signal. After A/D sampling at $44.1 \mathrm{kHz}$, the analog signal is transformed to digital signal. The specifications of the wideband FMCW radar system are listed in Table 1. Fig.3 shows the whole radar system.

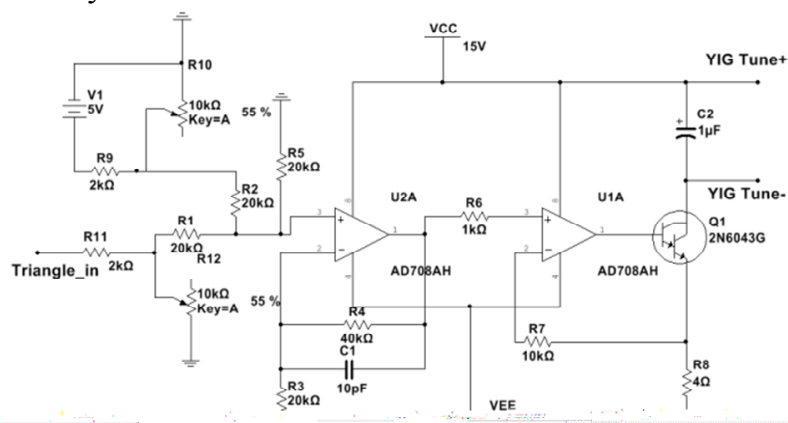

Fig. 2 Tuning circuit of YIG oscillator

\begin{tabular}{|c|c|c|c|}
\hline $\begin{array}{l}\text { Carrier } \\
\text { frequency }\end{array}$ & $10.16 \mathrm{GHz}$ & Bandwidth & $5.1 \mathrm{GHz}$ \\
\hline Sweep period & $0.207 \mathrm{~s}$ & Linearity & $2.2 \%$ \\
\hline $\begin{array}{l}\text { Transmitted } \\
\text { power }\end{array}$ & $16 \mathrm{dBm}$ & $\begin{array}{l}\text { Antenna } \\
\text { aperture }\end{array}$ & $0.14 \mathrm{~m}$ \\
\hline Rail length & $4 m$ & Speed & $0.101 \mathrm{~m} / \mathrm{s}$ \\
\hline
\end{tabular}

Table 1 Specifications of the wideband FMCW radar system

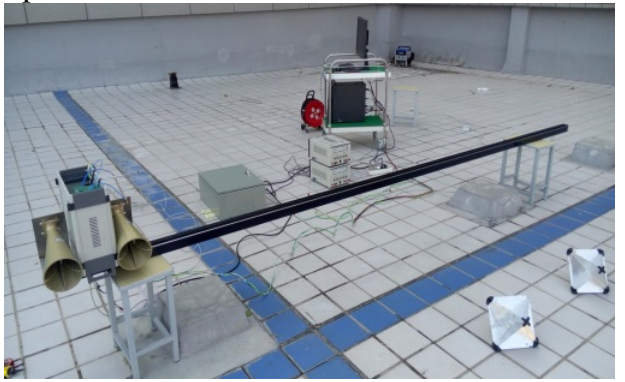

Fig. $3 \mathrm{X}$-band FMCW radar system with $5.1 \mathrm{GHz}$ bandwidth.

\section{Signal processing}

With a wide band, the nonlinearity of the transmitted signal can't be avoided. This leads to a deteriorated resolution by spreading a target's beat signal in a certain bandwidth, which can't meet the requirements of high quality imaging. In this section, a nonlinearity correction method based on HAF and the $\omega k$ imaging algorithm are demonstrated.

\subsection{Nonlinearity Correction}

Suppose that the transmitted signal is an LFM signal with the nonlinearity phase term $\varepsilon(t)$, which can be written as

$$
S_{t}(t)=\exp \left\{j\left[2 \pi\left(f_{c} t+\frac{1}{2} K_{r} t^{2}\right)+\varepsilon(t)\right]\right\}
$$

where $f_{c}$ is the initial frequency, $K_{r}$ is the linear chirp rate. The nonlinearity phase term $\varepsilon(t)$ can be expressed as a polynomial

$$
\varepsilon(t)=\sum_{k=3}^{K} b_{k-1} t^{k}
$$

where $K$ is often chosen from 4 to 7 . The reflected signal from $I$ targets is a sum of delayed and attenuated version of the transmitted signal

$$
S_{r}(t)=\sum_{i=1}^{I} \alpha_{i} \exp \left(j 2 \pi f_{c}\left(t-\tau_{i}\right)+j \pi K_{r}\left(t-\tau_{i}\right)^{2}+j 2 \pi \varepsilon\left(t-\tau_{i}\right)\right)
$$

where $\tau_{i}$ and $\alpha_{i}$ are the propagation delay and amplitude of target $i$.The dechirp operation mixes the received and transmitted signals to reduce the sampling rate, so that the beat signal is

$S_{\text {beat }}(t)=\sum_{i=1}^{I} \alpha_{i} \exp \left(j 2 \pi\left[-f_{c} \tau_{i}-K_{r} t \tau_{i}+\frac{1}{2} K_{r} \tau_{i}^{2}+\left(\varepsilon\left(t-\tau_{i}\right)-\varepsilon(t)\right)\right]\right)$

In (4), the first three exponential terms are the ideal beat signal, and the fourth term is the nonlinearity phase, which deteriorates target spectrum and range imaging quality. In short range applications, equation (4) can be approximated as

$$
S_{\text {beat }}(t)=\sum_{i=1}^{I} \alpha_{i} \exp \left(-j 2 \pi\left[f_{c}+K_{r} t+\varepsilon^{\prime}(t)\right] \tau_{i}\right)
$$

In this way, the beat signal is a multicomponent polynomial phase signal (PPS). The aim of nonlinearity correction is to estimate and eliminate the effect of $\varepsilon^{\prime}(t)$, which can be realized with HAF and time resampling.

Define a PPS as

$$
S_{P}(t)=A \exp \left(j 2 \pi \sum_{m=0}^{M} a_{m} t^{m}\right)
$$

Of which the high-order instantaneous momentum(HIM) is

$$
\operatorname{HIM}_{K}\left(S_{P}(t), \tau\right)=\prod_{k=0}^{K-1}\left[S_{P}{ }^{* k}(t-k \tau)\right]^{C_{K-1}^{k}}
$$

The definition of $S^{* k}(t)$ can be referred to [9]. The HAF is defined as the Fourier transform of the HIM. The property of the HIM is that the $M$ th-order HIM of $S_{P}(t)$ is reduced to a harmonic

$$
\operatorname{HIM}_{M}\left(S_{P}(t), \tau\right)=A^{2^{M-1}} \exp \left(j 2 \pi f_{M} t+\phi_{M}\right)
$$

where $\phi_{M}$ is constant phase term and

$$
f_{M}=M ! \tau^{M-1} a_{M}
$$


With the HAF, the spectral peak of HIM can be obtained to estimate the polynomial coefficients $a_{m}$ sequentially.

To get a well-focused range profile, nonlinearity correction should be conducted after the estimation of $\varepsilon^{\prime}(t)$. Rewrite (5) as

$$
S_{\text {beat }}(t)=\sum_{i=1}^{\infty} \alpha_{i} \exp \left(-j 2 \pi f_{c} \tau_{i}-j 2 \pi \hat{K}_{r}\left[t+\hat{\varepsilon}^{\prime}(t) / \hat{K}_{r}\right] \tau_{i}\right)
$$

Define a new time axis as

$$
t_{\text {interp }}=t+\varepsilon^{\prime}(t) / K_{r}
$$

and the corrected beat signal is

$$
S_{\text {beat }, \text { cor }}(t)=\sum_{i=1}^{\infty} \alpha_{i} \exp \left(-j 2 \pi f_{c} \tau_{i}-j 2 \pi \hat{K}_{r} t_{\text {interp }} \tau_{i}\right)
$$

Thanks to the new time axis, the beat signal becomes a sum of complex sinusoids. This new time axis can obtained through time domain interpolation. With this time resampling operation, the range-dependent nonlinearity can be corrected at a time.

\section{2 ${ }^{\omega k}$ Algorithm}

To validate the system design and validity of the nonlinearity correction method, rail-SAR imaging experiments are carried out. Under the conditions of large bandwidth and FMCW, the range walk during the pulse duration can't be neglected, so that a modified $\omega k$ algorithm is adopted.

The start point is the beat signal after the RVP has been removed

$$
S_{\text {beat }}\left(t^{\prime}, \tau\right)=\sigma \cdot \exp \left(-j 2 \pi f_{c} \tau-j 2 \pi K_{r} t^{\prime} \tau\right)
$$

Where $t^{\prime}$ is the range time variable and $\tau$ is the delay time defined as $\tau=[R(t+\tau)+R(t)] / c . R(t)$ is the instantaneous slant range of total time variable $t$, and we can calculate the delay time

$$
\tau=2 \delta\left[\frac{R(t)}{c}+\frac{v^{2}}{c^{2}}\left(\eta+t^{\prime}\right)\right]
$$

Where $\eta$ is the azimuth time variable and the "Doppler factor" $\delta$ is defined as $\delta=1 /\left(1-v^{2} / c^{2}\right)$. So, the beat signal can be written as

$S_{\text {beat }}\left(\eta, t^{\prime}, f_{r}\right)=\sigma \cdot \exp \left\{-j 4 \pi \delta\left(f_{c}+f_{r}\right) \times\left[\frac{R\left(\eta+t^{\prime}\right)}{c}+\frac{v^{2}}{c^{2}}\left(\eta+t^{\prime}\right)\right]\right\}$

Apply the FT with respect to the azimuth time variable $\eta$ to obtain the point target reference spectrum (PTRS). The phase of the spectrum is

$$
\Phi\left(f_{a}, f_{r}\right)=\frac{4 \pi \delta R_{0}}{c} \sqrt{\left(f_{c}+f_{r}\right)^{2}-\left[\frac{v}{c}\left(f_{c}+f_{r}\right)+\frac{c f_{a}}{2 \delta v}\right]^{2}}-2 \pi f_{a} \frac{f_{r}}{K}
$$

Compared to the pulsed SAR, the 2-D spectrum has additional range-azimuth coupling term and range walk term which are introduced by the variation of the slant range during the long pulse duration.

There are mainly two processing steps of the $\omega k$ algorithm.

Step1: Reference function multiplication (RFM) removes the range-invariant phase. The phase of the RFM filter can be expressed as
$\Phi_{R}\left(f_{a}, f_{r}\right)=\frac{4 \pi \delta R_{r e f}}{c} \sqrt{\left(f_{c}+f_{r}\right)^{2}-\left[\frac{v}{c}\left(f_{c}+f_{r}\right)+\frac{c f_{r}}{2 \delta v}\right]^{2}}-2 \pi f_{a} \frac{f_{r}}{K}(18)$

After RFM filtering, the phase of the remaining signal becomes

$\Phi_{R F M}\left(f_{a}, f_{r}\right)=\frac{4 \pi \delta\left(R_{0}-R_{r e f}\right)}{c} \sqrt{\left(f_{c}+f_{r}\right)^{2}-\left[\frac{v}{c}\left(f_{c}+f_{r}\right)+\frac{c f_{r}}{2 \delta v}\right]^{2}}$

Step2: Stolt interpolation removes all the phase terms higher than linear term, which also implements the residual azimuth phase and range-azimuth coupling. Stolt interpolation is given as

$$
\sqrt{\left(f_{c}+f_{r}\right)^{2}-\left[\frac{v}{c}\left(f_{c}+f_{r}\right)+\frac{c f_{r}}{2 \delta v}\right]^{2}} \rightarrow f_{c}+f_{r}^{\prime}
$$

After Stolt interpolation, the phase of the spectrum becomes

$$
\Phi_{\text {Stolt }}\left(f_{a}, f_{r}^{\prime}\right)=-\frac{4 \pi \delta\left(R_{0}-R_{r e f}\right)}{c}\left(f_{c}+f_{r}^{\prime}\right)
$$

At last, the SAR image can be obtained after 2-D IFFT to the spectrum.

\section{Experiment results}

In this section, some experiment results are given to demonstrate the performance of the wideband radar system and to validate the signal processing methods.

\subsection{Nonlinearity Correction Results}

To simulate the actual signal, a simulation was conducted under the specifications of the aforementioned wideband radar system. The nonlinearity of the transmitted signal was set $7.66 \%$ The bandwidth of the radar is $5 \mathrm{GHz}$. The period of the LFM signal is $100 \mathrm{~ms}$ and the signal is sampled by a personal computer with $44.1 \mathrm{kHz}$ sampling rate. Six targets were set in the scene at the location of $2 \mathrm{~m}, 5 \mathrm{~m}, 10 \mathrm{~m}, 15 \mathrm{~m}, 20 \mathrm{~m}$ and $25 \mathrm{~m}$. The amplitude of the six targets are same. The range profiles before and after nonlinearity correction were shown in Fig.4(a) and Fig.4(b).

As we can see in Fig.4(a), before nonlinearity correction, the targets' energy spreads in a certain bandwidth, which is more serious at large frequency. After nonlinearity correction, the six targets were well focused. Fig.4(b) is the detailed 22-28m range profile. Measurement of the target at $25 \mathrm{~m}$ shows that the peak level increases by about $15 \mathrm{~dB}$ and the $-3 \mathrm{~dB}$ resolution is $3 \mathrm{~cm}$, corresponding to the theoretical limit.

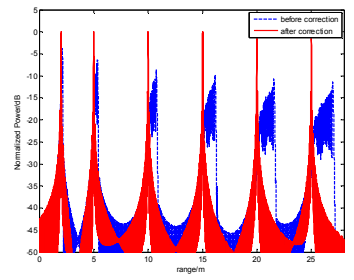

(a)

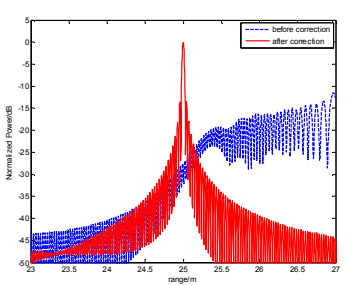

(b)
Fig. 4 Simulation results. (a) Six targets range profiles before and after nonlinearity. (b) Detailed $23-27 \mathrm{~m}$ range profile. Afterwards, a delay line about $4 \mathrm{~m}$ was employed to the radar to validate the correction method. The results are shown in Fig.5(a) and Fig.5(b). Fig.5(a) is the time domain signal and the corresponding spectrum is in Fig.5(b). Before nonlinearity 
correction, the range resolution is $3.01 \mathrm{~cm}$, greater than the theoretical limit. In addition, an enormous sidelobe exists in the left of main lobe. After correction, the range profile has remarkable improvement in performance, with the main lobe highlighted and the resolution enhanced to $2.84 \mathrm{~cm}$. The results have validated the correction method.

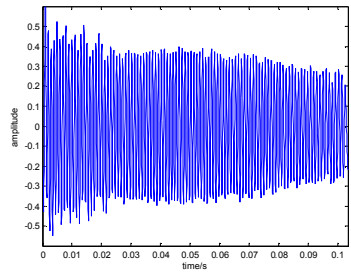

(a)

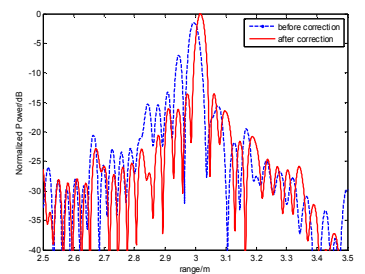

(b)
Fig. 5 Nonlinearity correction of a delay line. (a) Collected beat signal. (b) Detailed $2.5 \mathrm{~m}-3.5 \mathrm{~m}$ range profiles before and after nonlinearity.

\subsection{SAR images}

With the help of the $\omega k$ algorithm, we have conducted several detection experiments to demonstrate the performance of the wideband radar system. The FMCW radar was mounted on a $4 \mathrm{~m}$ rail and driven by a motor at $0.1 \mathrm{~m} / \mathrm{s}$. In Fig.6, a photograph of the copper cylinder and the corresponding SAR image are given. The copper cylinder has $2.5 \mathrm{~cm}$ height with $0.4 \mathrm{~cm}$ diameter. The image has a resolution of $3 \mathrm{~cm} \times 3 \mathrm{~cm}$, which can separate the copper cylinders easily.

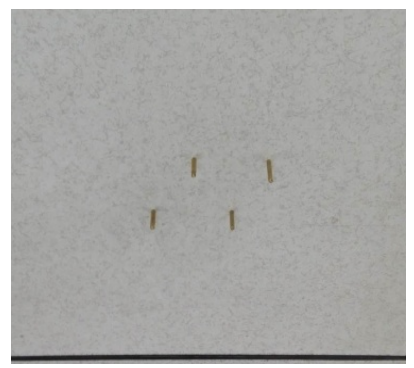

(a)

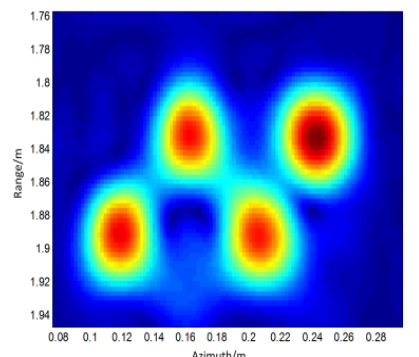

(b)
Fig. 6 Photograph of the copper cylinder and the corresponding SAR image. (a) Photograph. (b) SAR image.

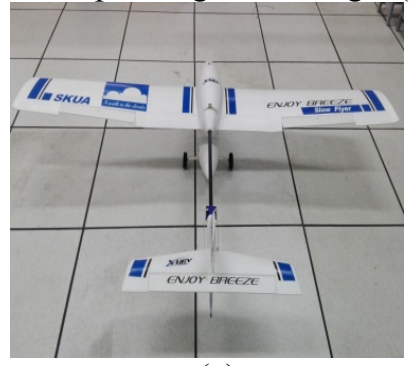

(a)

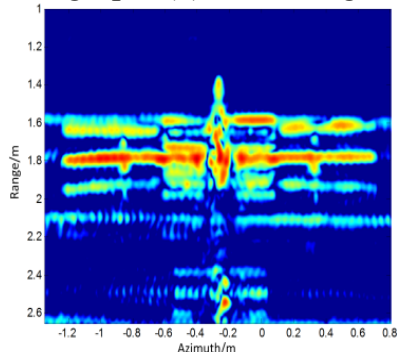

(b)
Fig. 7 Photograph of the model airplane and the corresponding SAR image. (a) Photograph. (b) SAR image. Fig.7 gives a photograph of the model airplane and the corresponding SAR image. In the image, the outline of the airplane can be seen clearly. In addition, the several aerofoils are caused by multiple effects.

At last, Fig.8 gives a photograph of the electric generator and the corresponding SAR image. The strong reflection is the Dihedral Angle formed by the metal surface. But the metal cavity has complex reflections.

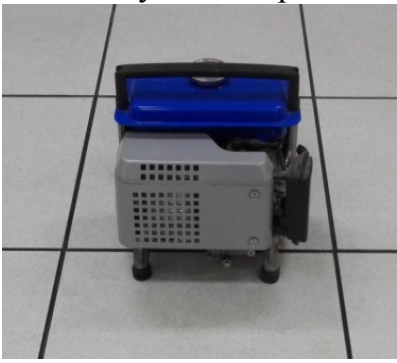

(a)

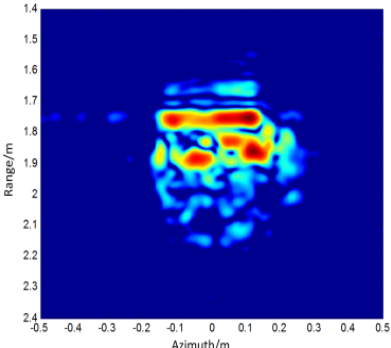

(b)
Fig. 8 Photograph of the electric generator and the corresponding SAR image. (a) Photograph. (b) SAR image.

\section{Conclusion}

In this paper, a wideband FMCW radar was demonstrated. The design of the transmitter and receiver were mainly introduced. In signal processing, the nonlinearity correction method and $\omega k$ imaging algorithm were employed. Range profiles and SAR images validated the performance of the radar system.

\section{References}

[1] Koh G, Lever J H, Arcone S A, et al. Autonomous FMCW radar survey of Antarctic shear zone[J]. International Conference on Ground Penetrating Radar, 2010:1-5.

[2] Justin Leigh Fernandes. Millimeter-Wave Imaging of Person-Borne Improvised Explosive Devices[D]. Northeastern University Boston, Massachusetts. Jan. 2010

[3] N. Pohl, T. Jacschke, and M. Vogt, "Ultra high resolution SAR imaging using an $80 \mathrm{GHz}$ FMCW-radar with 25 $\mathrm{GHz}$ bandwidth[C], in Proc. EuSAR, Nuremberg, Germany, Apr. 2012, pp. 189-192K. Elissa, "Title of paper if known," unpublished.

[4] Jaeschke, M. Uogt, C. Baer, C. Bredendiek, and N. Pohl, Improvements in distance measurement and SARimaging applications by using ultra-high resolution $\mathrm{mm}$ wave FMCW radar systems[C].Microwave Symposium Digest, 2012 IEEE MTT-S International, vol., no, pp.13, 17-22 June 2012.

[5] Yang Shang, Hao Y W, Chang Yang, Yuan Liang and Wei Meng Lim. A 239-281 GHz Sub-THz Imager with $100 \mathrm{MHz}$ Resolution by CMOS Direct-conversion Receiver with On-chip Circular-polarized SIW Antenna[C]. 978-1-4799-3286-3/14,2014 IEEE.

[6] A. Meta, P. Hoogeboom, and L. P. Ligthart. Signal processing for FMCW SAR[J], IEEE Trans. Geosci. Remote Sens. vol. 45, no. 11, Nov. 2007. pp. 3519-3532.

[7] Anghel A, Vasile G, Cacoveanu R, et al. Short-Range Wideband FMCW Radar for Millimetric Displacement Measurements[J]. IEEE Transactions on Geoscience \& Remote Sensing, 2014, 52(9) pp.5633 - 5642.

[8] Robert Wang,Otmar Loffeld,Holger Nies. Focus FMCW SAR Data Using the Wavenumber Domain Algorithm [J].IEEE TRANSACTIONS ON GEOSCIENCE AND REMOTE SENSING,48(4):2109-2117.

[9] S. Barbarossa, A. Scaglione, and G. B. Giannakis, Product high-order ambiguity function for multicomponent polynomial-phase signal modeling $[\mathrm{J}]$, IEEE Trans. Signal Process., vol. 46, no. 3, pp. 691-708. 